\title{
Velocity of shear waves in an initially stressed incompressible anisotropic medium
}

\author{
S DEY and P K DE \\ Department of Applied Mathematics, Indian School of Mines, \\ Dhanbad 826004, India \\ e-mail: cme@ismine.ernet.in
}

MS received 27 April 1998; revised 21 January 1999

\begin{abstract}
The paper aims at the study of propagation of shear waves in an initially stressed anisotropic medium. The velocity equation is obtained and it is seen that the velocity of propagation depends upon the direction of propagation, the anisotropic factor and also on the initial stresses. Numerical computation indicates that the initial compressive stress diminishes the velocity of a shear wave which propagates along the direction $0^{\circ}$ to $45^{\circ}$ but increases it along the direction $45^{\circ}$ to $90^{\circ}$. The reverse effect is obtained when initial stress is tensile. The anisotropy increases the velocity and even in the absence of initial stresses the velocity of the wave depends on the direction of propagation.
\end{abstract}

Keywords. Shear wave; initial stresses; anisotropy; incompressibility.

\section{Formulation}

Most materials behave as incompressible media and the velocities of longitudinal waves in them are very high. The varieties of hard rock present in the earth are also almost incompressible. Further, due to different factors such as the external pressure, slow process of creep, difference in temperature, manufacturing processes, nitriding, pointing etc. huge quantities of stresses (called initial stresses) are stored in the medium. Owing to the variation of elastic properties and due to the presence of these initial stresses the medium becomes anisotropic as well.

Consider an unbounded incompressible anisotropic medium under initial stresses $S_{22}$ and $S_{11}$ along the $y$ and $x$ directions respectively (figure 1). The equations of motion in the incremental state are (Biot 1965)

$$
\begin{aligned}
& \frac{\partial s_{11}}{\partial x}+\frac{\partial s_{12}}{\partial y}-P \frac{\partial \omega}{\partial y}=\rho \frac{\partial^{2} u}{\partial t^{2}} \\
& \frac{\partial s_{12}}{\partial x}+\frac{\partial s_{22}}{\partial y}-P \frac{\partial \omega}{\partial y}=\rho \frac{\partial^{2} v}{\partial t^{2}}
\end{aligned}
$$




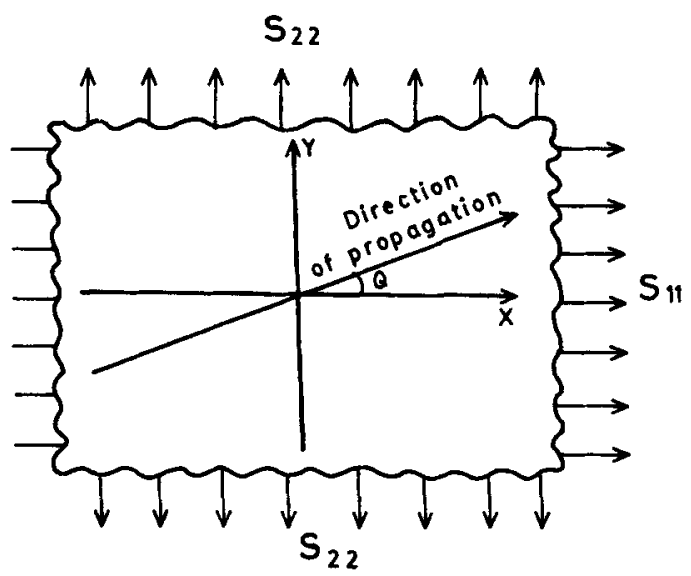

Figure 1. Geometry of the problem.

where $P=S_{22}-S_{11}$ and $\omega=\frac{1}{2}(\partial v / \partial x)-(\partial u / \partial \gamma), u, v$ being displacement components along $x$ and $y$ directions respectively, $\rho$ is the density of the medium and $s_{i j}$ are the incremental stresses. The stress-strain relations for an incompressible anisotropic medium may be taken as

where

$$
s_{11}-s=2 N e_{x x}, s_{22}-s=2 N e_{y y} \text { and } s_{12}=2 Q e_{x y},
$$

$$
s=\left(s_{11}+s_{22}\right) / 2 .
$$

The incompressibility condition $e_{x x}+e_{y y}=0$ is satisfied by

$$
u=\partial \phi / \partial x \text { and } v=\partial \phi / \partial y .
$$

\section{Solution}

Using (2) and (3) in (1) and then eliminating $s$ from these equations we get

$$
\begin{aligned}
& \left(Q+\frac{P}{2}\right) \frac{\partial^{4} \phi}{\partial x^{4}}+(4 N-2 Q) \frac{\partial^{4} \phi}{\partial x^{2} \partial y^{2}}+\left(Q-\frac{P}{2}\right) \frac{\partial^{4} \phi}{\partial y^{4}} \\
& =\rho\left[\frac{\partial^{4} \phi}{\partial x^{2} \partial t^{2}}+\frac{\partial^{4} \phi}{\partial y^{2} \partial t^{2}}\right] .
\end{aligned}
$$

For the propagation of waves in any arbitrary direction, one may take the solution of (4) as

$$
\phi(x, y, t)=A e^{i k\left(p_{1} x+p_{2} y-c t\right)},
$$

where $p_{1}$ and $p_{2}$ are the cosine of the angles made by the direction of propagation with the $x$ - and $y$-axis respectively and $c$ and $k$ are the velocity of propagation and the wave number respectively.

Using (5) in (4) the velocity of the shear wave propagated in the medium is obtained as

$$
\frac{c^{2}}{\beta^{2}}=2\left(2 \frac{N}{Q}-1\right) p_{1}^{2} p_{2}^{2}+\left(1-\frac{P}{2 Q}\right) p_{1}^{4}+\left(1+\frac{P}{2 Q}\right) p_{2}^{4}
$$


where $\beta=(Q / \rho)^{1 / 2}$, the velocity of the shear wave in an initially stress-free isotropic medium. Equation (6) shows that the velocity $(c / \beta)$ depends much on the anisotropy factor $N / Q$, the initial stress factor $P / 2 Q$ and also the direction of propagation given by $p_{1}$ and $p_{2}$. The particular cases discussed in the next section may be of interest.

\section{Particular cases}

(1) When $N=Q$, (6) on simplification gives

$$
\frac{c^{2}}{\beta^{2}}=1+\frac{P}{2 Q}\left(p_{2}^{2}-p_{1}^{2}\right)
$$

This shows that along a direction $45^{\circ}$ to the $x$-axis, velocity of propagation is independent of the initial stresses. However, this is not so in the case of anisotropy. In case $S_{22}-S_{11}=0$ or $S_{22}=S_{11}$, then $P=0$ and $c=\beta$, i.e. in an isotropic medium, the hydrostatic initial stresses have no effect on the propagation of shear waves.

(2) When $P=0$, but the material is anisotropic, then the velocity is given by

$$
c^{2} / \beta^{2}=1+4(N / Q-1) p_{1}^{2} p_{2}^{2} .
$$

This shows that velocity-propagation is dependent on the direction of propagation and is maximum along a direction $45^{\circ}$ to the $x$-axis, if $N / Q>1$, and is minimum when $N / Q<1$.

(3) The velocity of propagation along the $x$ - and $y$-axis when $P=0$ is given by $c=\beta$, and along a direction $45^{\circ}$ to them is $c / \beta=(N / Q)^{1 / 2}$.

(4) The velocity of propagation in an initially stressed anisotropic medium along the $x$ direction from (6) is given by $c_{x} / \beta=(1-P / 2 Q)^{1 / 2}$. Since $P=S_{22}-S_{11}$ the effect of initial stresses is compressive along the $x$ direction if $P$ is positive; in that case $c_{x}<\beta$, but if $P<0$, then the effect of initial stress along the $x$-direction is tensile and $c_{x}>\beta$.

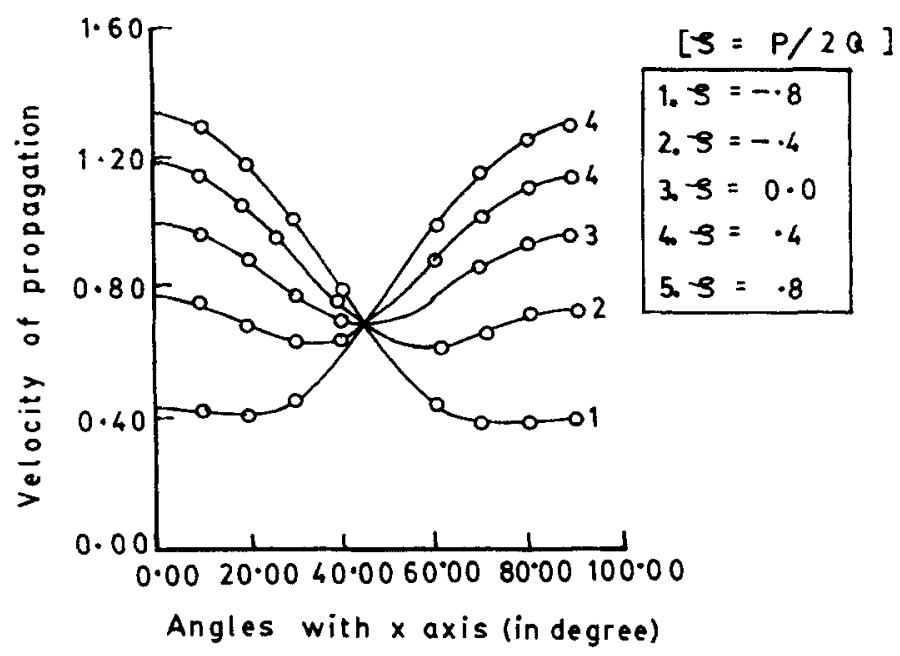

Figure 2. Variation of velocity of propagation with direction for $N / Q=0.5$. 


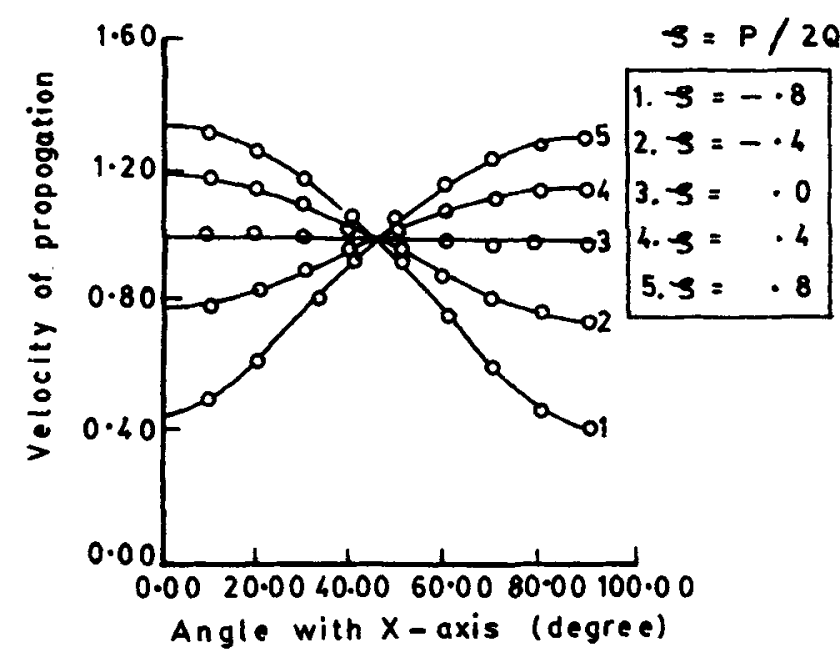

Figure 3. Variation of velocity of propagation with direction for isotropic medium $(N / Q=1)$.

(5) The velocity along the $y$-direction is $c / \beta=(1+P / 2 Q)^{1 / 2}$ which increases as $P$ increases and $c_{y}>\beta$, if $P$ is compressive and $c_{y}<\beta$ in case $P$ is tensile.

Further, these results give $c^{2}{ }_{y}-c^{2}{ }_{x}=P / \rho$. These show that the difference of squares of velocities along the $y$ and $x$ directions is a function of the initial stresses and density only and is independent of the elastic constants. Since $P=S_{22}-S_{11}$, in case $S_{22}>S_{11}$, then $c_{y}>c_{x}$; the reverse is the case when $S_{22}<S_{11}$. If $S_{22}$ is positive (tensile stress) and $S_{11}$ is negative (compressive stress), then $c_{y}>c_{x}$.

\subsection{Numerical computations and discussion}

Numerical values of velocity of propagation in an incompressible medium have been computed for some admissible values of $P / 2 Q$ and $N / Q$ and are presented in figures 2 to 6 . Figure 2 presents the values of $c / \beta$ for different values of $P / 2 Q$, when $N / Q=5$. Figure 3

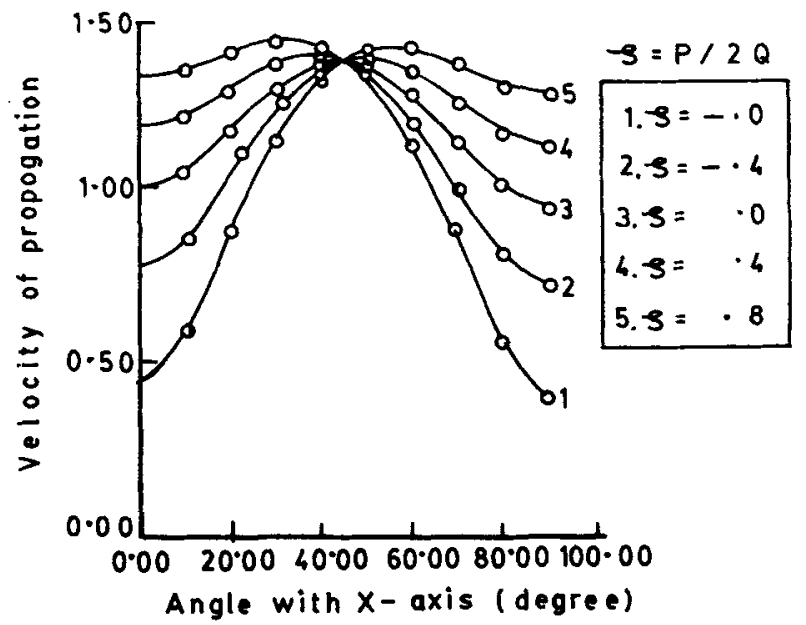

Figure 4. Variation of velocity of propagation with direction for anisotropic medium $(N / Q=2)$. 


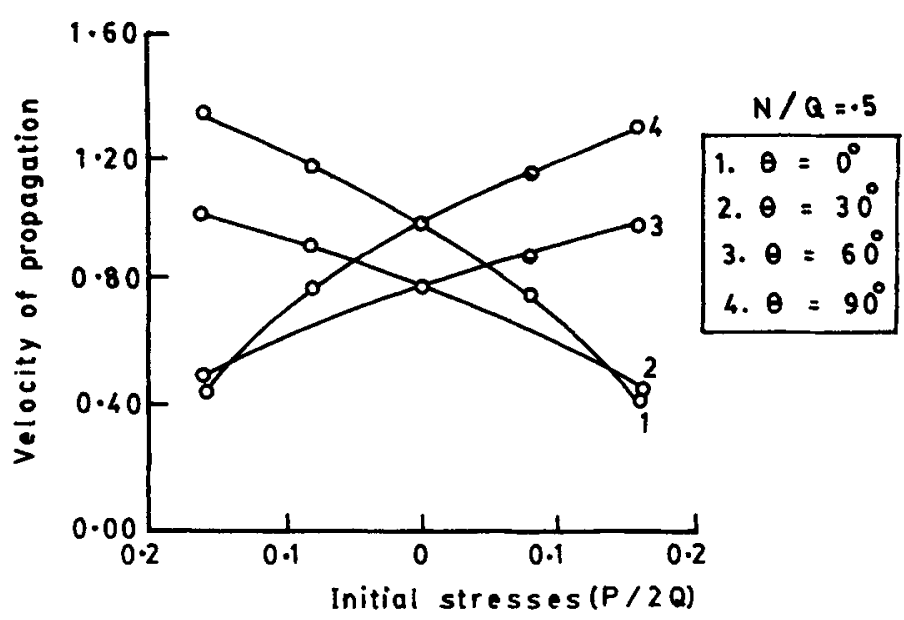

Figure 5. Variation of velocity of propagation with respect to $\zeta$ for different directions of propagation, when $N / Q=0.5$.

shows the same for an isotropic case $N / Q=1$, whereas figure 4 represents $N / Q=2$. These figures show that tensile stress $(P / 2 Q=\zeta=$ negative) decreases the velocity of propagation whereas the compressive stress $(\zeta=$ positive) increases it as the angle of the direction of propagation with the $x$-axis increases. In the absence of initial stress $(\zeta=0)$ the velocity of propagation is independent of direction in an isotropic medium, whereas the velocity depends on anisotropic factor $(N / Q)$. In addition, the velocity of propagation is different in different directions of propagation in the anisotropic medium in the presence or absence of initial stress. In case $N / Q$ is less than 1 , the velocity decreases as the directional angle increases and becomes minimum at $45^{\circ}$. In the case when $N / Q>1$, the reverse effect is observed.

The effect of the presence of initial stresses has also been shown in the curves of figures 5 and 6 . The variation of velocity of propagation with respect to direction of propagation at different anisotropic parameters have been given in figure 7. It is observed that as anisotropy increases the velocity also increases. Figures 7 and 8 have been given in order to show the effect of presence of initial stresses and increasing anisotropy.

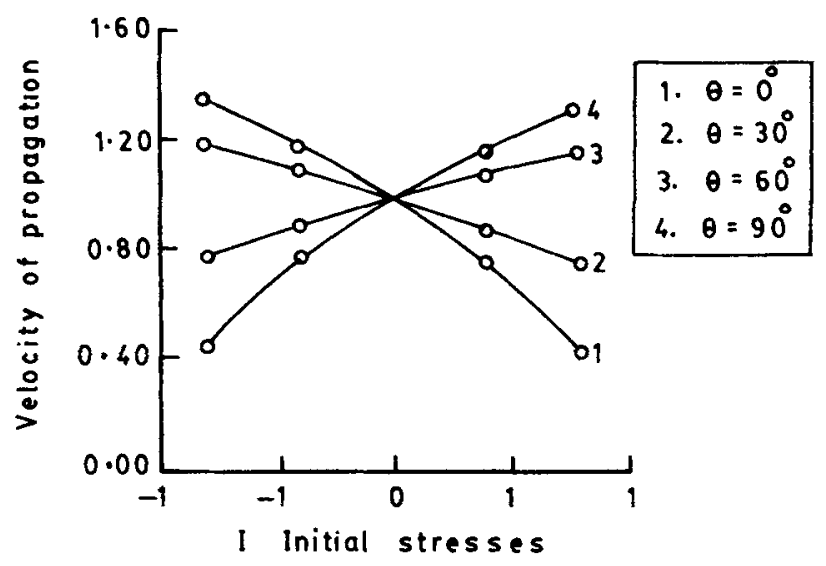

Figure 6. Variation of velocity of propagation with respect to $\zeta$ for different directions of propagation, when $N / Q=1$. 

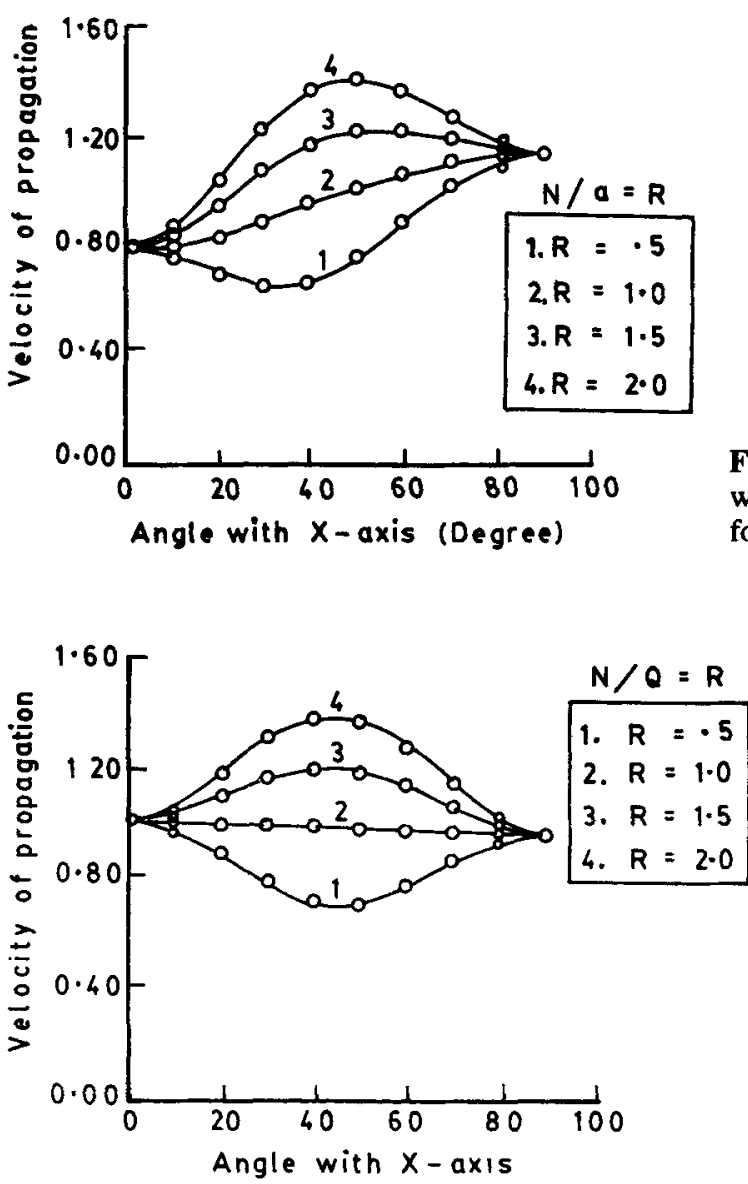

Figure 7. Variation of velocity of propagation with respect to angle of propagation with $\mathrm{X}$-axis for $\zeta=0.4$.
Figure 8. Variation of velocity of propagation with respect to angle of propagation with $\mathrm{X}$-axis for $\zeta=0.0$.

\section{Conclusions}

The study reveals that the presence of initial stresses and anisotropy plays an important role in the velocity of propagation of waves in a medium. The incompressibility of the medium has been taken into account only to remove the complexity in calculation. The propagation of waves may also be studied for compressible media where longitudinal waves also appear. The anisotropic factor as well as the initial stress parameter make the velocity of propagation depend on the direction of propagation. Increase of the value of $N / Q$ increases the velocity. The compressive initial stress along the direction of propagation increases the velocity of propagation of shear waves when the direction of propagation is upto $45^{\circ}$ with the direction of the initial stress, after which the velocity decreases. The reverse effect is observed in case of initial tensile stress.

\section{References}

Biot M A 1965 Mechanics of incremental deformations (New York: John Wiley and Sons) 